\title{
Exercícios Orientais Terapêuticos: Impacto sobre biomarcadores de estresse e contribuições a estudantes universitários
}

\author{
Oriental Therapeutic Exercises: Impact on stress biomarkers and contributions to university
} students

Ejercicios Terapéuticos Orientales: Impacto en biomarcadores de estrés y aportes a estudiantes universitarios

Suellen Andrade Pereira ORCID: https://orcid.org/0000-0003-2485-349X Universidade Federal de Alfenas, Brasil E-mail: suellen.pereira@sou.unifal-mg.edu.br Vânia Regina Bressan ORCID: https://orcid.org/0000-0003-2227-2755 Universidade Federal de Alfenas, Brasil E-mail: vania.bressan@unifal-mg.edu.br Adriana Teresa Silva Santos ORCID: https://orcid.org/0000-0002-9959-3269 Universidade Federal de Alfenas, Brasil E-mail: adriana.santos@unifal-mg.edu.br

Lucas Fernandes Feres ORCID: https://orcid.org/0000-0003-2345-3574 Universidade Federal de Alfenas, Brasil

E-mail: lucas.feres@ sou.unifal-mg.edu.br Isabelle Cristinne Pinto Costa ORCID: https://orcid.org/0000-0002-2611-8643 Universidade Federal de Alfenas, Brasil

E-mail: isabelle.costa@unifal-mg.edu.br

Simone Albino da Silva

ORCID: https://orcid.org/0000-0002-2725-8832 Universidade Federal de Alfenas, Brasil E-mail: simone.silva@unifal-mg.du.br

Andréia Maria Silva Vilela Terra

ORCID: https://orcid.org/0000-0002-1255-8429 Universidade Federal de Alfenas, Brasil E-mail: andreia.silva@unifal-mg.edu.br João Batista Camargos Júnior

ORCID: https://orcid.org/0000-0001-5510-5748 Universidade Federal de Alfenas, Brasil E-mail: jbcamargos_18@hotmail.com

\begin{abstract}
Resumo
Objetivo: Avaliar o impacto de Exercícios Orientais Terapêuticos sobre biomarcadores de estresse e contribuições a estudantes universitários. Método: Estudo misto explanatório sequencial desenvolvido com 19 estudantes universitários entre outubro e dezembro de 2019. Foram coletados dados sociodemográficos, medidas de alfa amilase salivar e variabilidade da frequência cardíaca antes e após os exercícios e realizado grupo focal numa única sessão. Resultados: A maioria era mulher, média de 22 anos, cor branca, não heterossexual, sem religião e filhos, não exerciam atividade remunerada, residiam em repúblicas, renda mensal familiar bruta de até dois salários mínimos e dependiam financeiramente da família. Eram os primeiros membros da família a entrar em universidade pública, assistidos pelo programa de assistência estudantil, na fase final de cursos integrais e satisfeitos com a escolha. A prática Exercícios Orientais Terapêuticos influenciou no aumento da variabilidade da frequência cardíaca, auxiliando no relaxamento. Houve contribuições para a promoção da saúde e na construção de rede de apoio social. Conclusão: Há demanda para ações institucionais, como a oferta de práticas integrativas e complementares, que favoreçam a promoção da saúde e o apoio social dos estudantes. A prática estudada compreende um método inovador norteado pela integralidade e necessidades ampliadas de saúde dos estudantes universitários.
\end{abstract}

Palavras-chave: Terapias complementares; Estudantes; Promoção da saúde; Apoio social; Biomarcadores. 


\begin{abstract}
Objective: To evaluate the impact of Oriental Therapeutic Exercises on stress biomarkers and contributions to university students. Method: Mixed sequential explanatory study carried out with 19 university students between and December 2019. Sociodemographic data, measurements of salivary alpha amylase and heart rate variability were collected before and after the exercises and a focus group was performed in a single session. Results: The majority were women, average of 22 years old, white, non-heterosexual, without religion and children, did not exercise paid activity, lived in republics, gross monthly family income of two prices and depended financially on the family. They were the first members of the family to enter a public university, assisted by the student assistance program, in the final stage of integral courses and scoring with the choice. The practice of Oriental Therapeutic Exercises influenced the increase in heart rate variability, helping to relax. Contributions to health promotion and the construction of a social support network. Conclusion: There is a demand for institutional actions, such as an offer of integrative and complementary practices, which favor the promotion of health and social support of students. The studied practice comprises an innovative method guided by the comprehensiveness and expanded health needs of university students.

Keywords: Complementary therapies; Students; Health promotion; Social support; Biomarkers.
\end{abstract}

\title{
Resumen
}

Objetivo: Evaluar el impacto de los Ejercicios Terapéuticos Orientales sobre los biomarcadores de estrés y las contribuciones a los estudiantes universitarios. Método: Estudio explicativo secuencial mixto realizado con 19 estudiantes universitarios entre y diciembre de 2019. Se recolectaron datos sociodemográficos, medidas de alfa amilasa salival y variabilidad de la frecuencia cardíaca antes y después de los ejercicios y se realizó un grupo focal en una sola sesión. Resultados: La mayoría eran mujeres, promedio de 22 años, blancas, no heterosexuales, sin religión e hijos, no ejercían actividad remunerada, vivían en repúblicas, renta familiar mensual bruta de dos precios y dependían económicamente de la familia. Fueron los primeros miembros de la familia en ingresar a una universidad pública, asistidos por el programa de asistencia al estudiante, en la etapa final de los cursos integrales y puntuándose con la elección. La práctica de Ejercicios Terapéuticos Orientales influyó en el aumento de la variabilidad de la frecuencia cardíaca, contribuyendo a la relajación. Contribuciones a la promoción de la salud y la construcción de una red de apoyo social. Conclusión: Existe una demanda de acciones institucionales, como una oferta de prácticas integradoras y complementarias, que favorezcan la promoción de la salud y el apoyo social de los estudiantes. La práctica estudiada comprende un método innovador guiado por la integralidad y las necesidades de salud ampliadas de los estudiantes universitarios.

Palabras clave: Terapias complementarias; Estudiantes; Promoción de la salud; Apoyo social; Biomarcadores.

\section{Introdução}

As manifestações fisiológicas do organismo frente ao estresse ocorrem por atuação do Sistema Nervoso Autônomo (SNA) que ao promover a liberação de mediadores químicos provoca o aumento da frequência cardíaca e da pressão arterial pela estimulação simpática, além da secreção da enzima alfa amilase salivar (AAS). A ativação excessiva ou inadequada da função autonômica acarreta prejuízo à saúde e menor variabilidade da frequência cardíaca (VFC) (Mcewen, 2007; Ali \& Nater, 2020).

Evidencia-se que a presença de estresse entre os estudantes universitários coloca em risco sua saúde física e mental, ao provocar desde alteração nos hábitos de vida até no estado geral de saúde (Oliveira et al., 2020). Em 2018, a falta de disciplina e de hábitos de estudos, dificuldades financeiras, demanda excessiva de trabalhos estudantis e problemas emocionais, foram as principais dificuldades que impactaram o desempenho acadêmico dos estudantes das Instituições Federais de Ensino Superior (IFES). Entre os 84\% dos estudantes que relataram dificuldades emocionais, a ansiedade, o desânimo ou a desmotivação foram as mais citadas (Andifes, 2019).

Outro aspecto importante é o suicídio, considerado um problema de saúde pública com aumento considerável entre estudantes universitários (Santos et al., 2017). O percentual de ideação de morte e de pensamento suicida nas IFES cresceu aproximadamente $40 \%$ de 2016 para 2018 (Andifes, 2019).

Diante desse contexto, em 2018 surgiu o Programa de Extensão Unifal Sem Estresse (PULSE). Dentre as atividades oferecidas pelo PULSE, estão os Exercícios Orientais Terapêuticos (EOT), conduzidos por um instrutor capacitado e idealizador dessa prática, em parceria com o Programa Cidade Escola da Prefeitura Municipal. EOT é uma prática que envolve técnicas orientais físicas e mentais da Medicina Tradicional Chinesa (MTC), sendo elas: o do-in, o shiatsu, a yoga e a 
meditação (Brasil, 2018). E ainda a prática de artes marciais de origem chinesa (Kung Fu) (Rocha, 2005).

A MTC consiste em um sistema médico integral chinês de origem milenar que descreve, através da sua cosmologia, a inter-relação harmônica entre o macro e o microcosmos. Entende-se cosmologia como a forma que uma sociedade concebe a criação do universo formando sistemas que atribuem sentido a sua criação e existência. Posto que o adoecimento é um processo contínuo, o diagnóstico chinês busca identificar a desarmonia da circulação do Qì pelo corpo, que corresponde à energia vital e, assim, antecipar a evolução de enfermidades (Coutinho \& Dulcetti, 2015).

O raciocínio utilizado na MTC é estruturado pela teoria Yin e Yang, compreendida como duas forças opostas e complementares que compõem todos os fenômenos, movimentam o ciclo da natureza e a vida. Como também, a teoria dos cinco elementos (madeira, fogo, terra, metal e água) representados por um pentagrama que se influenciam mutuamente e cada elemento possui um tipo de $Q i$, um órgão, uma víscera, um sentimento, um sabor, correspondentes à essência manifestada na natureza desse elemento. (Coutinho \& Dulcetti, 2015; Brasil, 2018). Na superfície corporal existem pontos sensíveis conectados em sequência relacionados a função de um órgão interno denominados meridianos (Wen, 2008). Para que o sujeito esteja saudável, o Qì precisa circular harmonicamente no corpo pelos meridianos energéticos (Coutinho \& Dulcetti, 2015).

Em 1992, iniciou-se um processo que reconhece outras racionalidades médicas (RM) que explicam e intervém no processo saúde-doença, orientados por uma cosmologia diferente da medicina hegemônica ocidental, denominada biomédica. Assim, possibilitou diferenciar as racionalidades médicas de outras terapias, como as Práticas Integrativas e Complementares (PICs) e as outras Medicinas Tradicionais fundamentadas em uma matriz tradicional mais ampla não contemplada pela medicina convencional (Tesser, 2009; Nascimento et al., 2013).

No Brasil, as PICs foram incorporadas no Sistema Único de Saúde (SUS) em 2006 por meio da Política Nacional de Práticas Integrativas e Complementares (PNPIC) (Brasil, 2006). A PNPIC busca atender recomendações da Organização Mundial da Saúde (OMS) quanto a necessidade de apoiar e implementar práticas não medicamentosas seguras e eficazes para estimular mecanismos naturais de prevenção de agravos e de recuperação da saúde, bem como a ampliação de cuidados em saúde de forma integral e holística, complementando os tratamentos convencionais (Brasil, 2018).

Ressalta-se a importância de ações integradas, como a utilização das PICs e da cosmologia chinesa, ou seja, o seu entendimento sobre o processo saúde e adoecimento baseado nos cinco elementos, nos meridianos energéticos e na teoria do yin e yang, de forma a auxiliar os estudantes no enfrentamento das experiências causadoras de estresse e escolha de comportamentos mais saudáveis que impliquem positivamente na qualidade de vida e saúde. Desse modo, busca-se com este estudo, avaliar o impacto da EOT sobre biomarcadores de estresse e suas contribuições a estudantes universitários.

\section{Metodologia}

\section{Aspectos éticos}

A pesquisa foi aprovada pelo Comitê de Ética em Pesquisa da universidade, sob o n ${ }^{\circ}:$ 20397019.7.0000.514.

\section{Tipo de estudo}

Estudo misto com a estratégia explanatória sequencial (Creswell \& Plano Clark, 2018). Na primeira etapa foi realizado um estudo quantitativo de intervenção quase experimental, sem grupo controle do tipo pré/pós teste e na sequência foi realizada uma pesquisa qualitativa através de um grupo focal utilizando a análise de conteúdo (Bardin, 2016).

Os métodos mistos possibilitam a compreensão acerca das contradições entre achados quantitativos e qualitativos. O desenho explanatório sequencial busca explicar os achados quantitativos em uma segunda fase qualitativa (Creswell \& Plano Clark, 2018). Um estudo de intervenção avalia previamente e posteriormente o efeito de uma determinada intervenção. No delineamento quase-experimental, a pesquisa é desenvolvida com grupo único de indivíduos sem randomização (Polit \& Beck, 
2018).

\section{População, amostra e participantes}

Utilizou-se a amostragem em sequência, a qual recruta todos os estudantes da população que atendam aos requisitos de elegibilidade durante um intervalo de tempo específico (Polit \& Beck, 2018).

Os critérios de inclusão adotados foram estudantes dos cursos de graduação presenciais, com idade igual ou superior a 18 anos que participaram da EOT por um período igual ou superior a três meses. Utilizou-se como critérios de exclusão a impossibilidade de responder ao questionário sociodemográfico, a prática de atividade física intensa e a ingesta de substâncias como café, álcool, cafeína, nicotina, refrigerantes, energéticos na véspera e no dia da coleta de dados, que pudessem alterar a VFC (Catai et al., 2020).

Assinala-se que 25 participantes da EOT estavam dentro dos critérios de inclusão supracitados. Contudo, a amostra foi composta por 19 estudantes na primeira etapa de coleta de dados e 15 na segunda etapa. Esses foram os estudantes que puderam comparecer nos dias estabelecidos para a coleta de dados das duas etapas, os quais ocorreram no período de outubro a dezembro de 2019.

\section{Procedimentos e instrumentos de coleta dos dados \\ Pré-intervenção}

$\mathrm{Na}$ primeira etapa, a quantitativa, as variáveis fisiológicas (VFC e AAS) foram coletadas em dois momentos do mesmo dia, no período da tarde (antes e após a intervenção, ou seja, a EOT).

Os estudantes permaneceram em silêncio e repouso na posição de decúbito dorsal durante vinte minutos, para verificação da VFC nos cinco minutos finais pelo cardiofrequencímetro Polar v800 (Figura 1), que é um dos instrumentos amplamente utilizado para obter dados que permitam analisar a VFC. Trata-se de um instrumento não invasivo, seguro e que oferece praticidade na utilização em qualquer ambiente (Lopes et al., 2014).

Figura 1 - Posicionamento da cinta torácica.

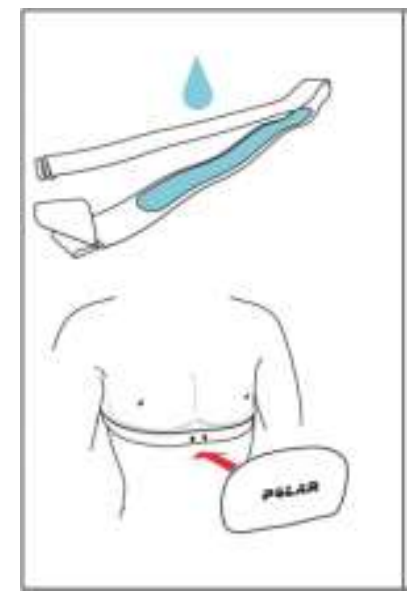

Fonte: User Manual for Polar V88 in English (2019).

Paralelamente, por meio de uma fita de teste descartável posicionada na região sublingual do participante durante 30 segundos foram mensurados os níveis de AAS por um leitor portátil Cocoro Meter (Nipro Co., Osaka, Japan) (Figura 2). Nesse estudo considerou-se a presença de estresse a média acima de $34 \mathrm{KU} / \mathrm{l}$ de AAS (Yamaguchi et al., 2004). Este instrumento é válido e preciso para análise da AAS como marcador de estresse psicológico (Shetty et al., 2011; Yamaguchi et al., 2004). 
Figura 2 - Fita de teste salivar e analisador salivar portátil.

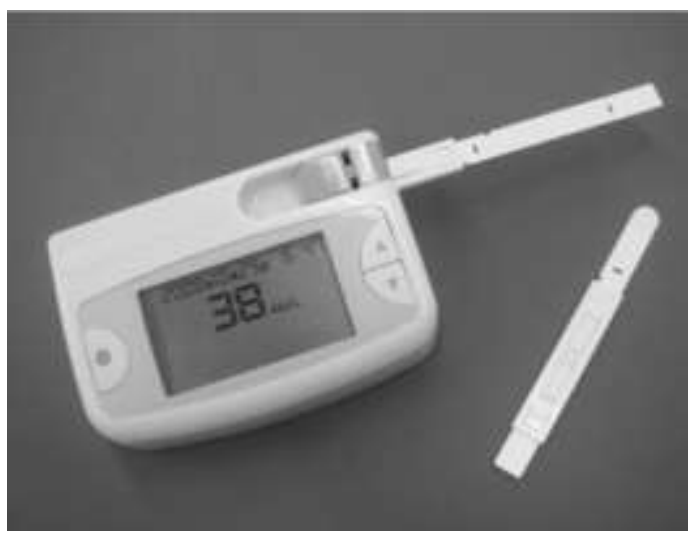

Fonte: Shetty et al., (2011)

A coleta de, no máximo, cinco participantes por dia, foi realizada por dois pesquisadores previamente treinados. Nesta etapa também foram coletados dados sociodemográficos na plataforma Google Forms, por meio de um questionário elaborado pelas pesquisadoras e refinado por pedagogos e psicólogos da instituição que prestam atendimento aos estudantes, que testaram sua viabilidade.

\section{Intervenção}

A intervenção foi desenvolvida em cinco tempos: $1^{\circ}$ ) duração de dez minutos para realização de exercícios aeróbicos de aquecimento; $2^{\circ}$ ) quinze minutos para a execução de posições da yoga relacionadas ao elemento (teoria dos 5 elementos) que constituiu o tema da aula; $3^{\circ}$ ) dez minutos nos quais se trabalhou o Do-in (Figura 3), com orientação ativa ou passiva alinhado ao tema da aula com a técnica de massagem chinesa shiatsu; $4^{\circ}$ ) quinze minutos para o desenvolvimento dos katis do kung fu (Figura 4); $5^{\circ}$ ) dez minutos para a prática de meditação passiva. O tempo total da intervenção foi de 60 minutos.

Figura 3 - Pontos do do-in/acupressão adaptados aos elementos de acordo com os meridianos dos órgãos e vísceras ao qual pertencem. HT - Coração; ID - Intestino Delgado; SP - Baço/Pâncreas; ST- Estômago; LU - Intestino Grosso; LI - Pulmão; KI - Rim; BL - Bexiga; LR - Fígado; GB - Vesícula Biliar.

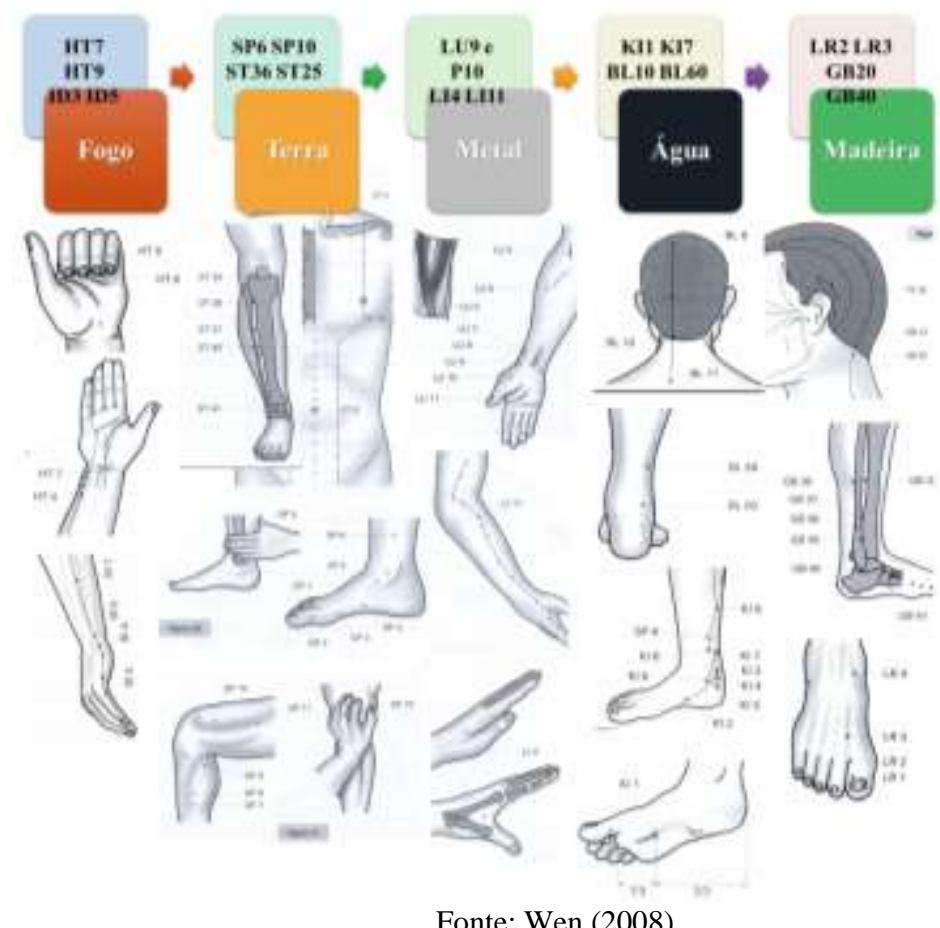

Fonte: Wen (2008) 
Figura 4 - Katis do kungfu.

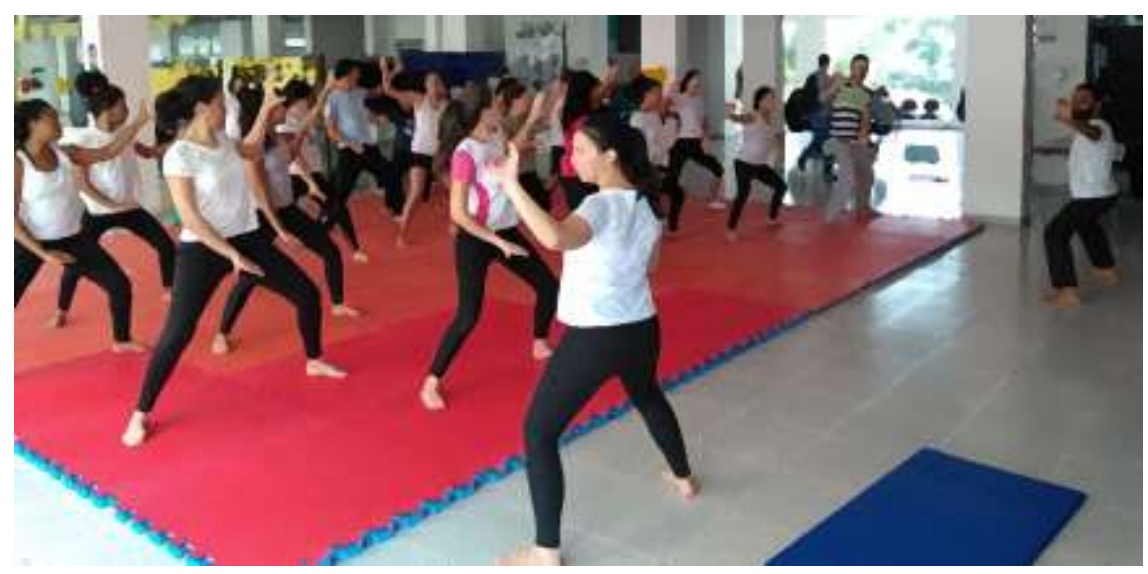

Fonte: Resende (2019).

\section{Pós-Intervenção}

Após a intervenção, os parâmetros fisiológicos foram coletados novamente e, em seguida, todos os participantes foram convidados a participar do grupo focal que compôs a etapa qualitativa. Este foi realizado em sessão única conduzido pela enfermeira e docente da Escola de Enfermagem da instituição de estudo com duração de uma hora e meia. Utilizou-se questões norteadoras semiestruturadas sobre a opinião dos estudantes em relação às contribuições da EOT no âmbito acadêmico e pessoal e como se sentiam após a prática. Os depoimentos foram gravados em aparelho de voz digital e transcritos na íntegra. Para a identificação e a preservação do anonimato dos participantes, utilizou-se como código nomes populares de plantas.

O estudo qualitativo compreende a intensidade dos fenômenos, trabalha com enfoque para a dimensão sociocultural expressa por meio de crenças, valores, opiniões, representações, formas de relação, simbologia, costumes, comportamentos, práticas e pensamentos da população estudada sobre o objeto de estudo (Minayo, 2017). Além disso, os grupos focais têm se mostrado uma estratégia útil de coleta de dados para pesquisas que tem como proposta a compreensão de experiências grupais e transformação da realidade (Kinalski et al., 2017).

\section{Tratamento e análise dos dados}

As informações foram tabuladas em planilhas do Microsoft Office Excel (2016). Os dados foram analisados por estatística descritiva, utilizando distribuições de frequências e porcentagens. Para a análise estatística das medidas fisiológicas, a normalidade dos dados foi avaliada pelo teste Shapiro-Wilk, posteriormente aplicou-se o teste de Wilcoxon para verificar a diferença antes e após a intervenção realizada.

As variáveis da VFC foram submetidas ao Software Kubios HRV Standard (versão 3.1.0.1), o qual forneceu os resultados de VFC no Domínio Tempo e Frequência. As variáveis foram associadas pelo teste qui-quadrado ou Exato de Fisher. Os dados foram analisados pelo programa estatístico Statistical Package for the Social Sciences (SPSS) versão 20.0, considerando nível de significância de $\mathrm{p}<0,05$.

O conteúdo dos depoimentos foi analisado qualitativamente por meio da técnica de análise de conteúdo temático propostos por Bardin (2016): pré-análise (leitura dos discursos para apreensão das ideias principais emergidas, em consonância com o objetivo do estudo), exploração do material (trechos dos discursos dotados de significado -núcleos de sentido- foram destacados e separados para a realização da categorização), tratamento dos resultados (agrupamento das unidades de significado que constituirão as categorias temáticas), inferência e interpretação dos dados (respaldadas no referencial teórico acerca da temática). 


\section{Resultados}

\section{Perfil sociodemográfico dos participantes}

Identificou-se que a maioria $(73,7 \%)$ era mulher, com média de 22 anos, de cor branca (37\%), orientação sexual como bissexual, homossexual, pansexual e assexual (52,6\%); sem religião $(52,6 \%)$ e sem filhos. Não exerciam atividade remunerada (78,9\%), residiam em repúblicas $(84,2 \%)$, possuíam renda mensal familiar bruta de até dois salários mínimos $(73,7 \%)$ e dependiam financeiramente da família $(84,2 \%)$. A maior parte foi o primeiro membro da família a entrar em universidade pública (58\%) e o primeiro da geração a ingressar numa universidade (31,6\%). Eram assistidos pelo programa de assistência estudantil (57,9\%), pertenciam a cursos de turno integral $(42,1 \%)$, estavam na fase final do curso $(57,9 \%)$ e satisfeitos com a escolha $(63,2 \%)$. Praticavam atividade física duas a três vezes na semana $(57,9 \%)$, ingeriam bebidas alcoólicas pelo menos uma vez na semana $(52,5 \%)$, dormiam de sete a oito horas por dia $(68,4 \%)$ e não eram tabagistas $(63,2 \%)$.

\section{Biomarcadores de estresse}

A Tabela 1 demonstra a análise da AAS, da VFC pré e pós intervenção e a correlação das variáveis. Verificou-se um aumento significativo da variável $R-R(p=0,04)$ e que não houve relevância estatística nos demais parâmetros, incluindo a associação entre AAS e as variáveis R-R e LF/HF.

Tabela 1 - Análise da $\alpha$-amilase salivar, variabilidade da frequência cardíaca dos participantes $(n=19)$ pré e pós intervenção, correlação entre as variáveis. Alfenas, MG, Brasil, 2020.

\begin{tabular}{|c|c|c|c|}
\hline \multirow[t]{2}{*}{ Variáveis } & \multicolumn{3}{|c|}{$\begin{array}{c}\text { Participantes } \\
(\mathrm{n}=19)\end{array}$} \\
\hline & Pré Intervenção & Pós Intervenção & $\mathrm{p}$ \\
\hline \multirow{2}{*}{$\begin{array}{l}\text { AAS (KU/L) - } \\
\text { Média } \pm \text { DP } \\
\text { IC 95\% }\end{array}$} & $29,89 \pm 21,26$ & $28,36 \pm 25,39$ & \multirow[b]{2}{*}{0,77} \\
\hline & $19,64-40,14$ & $16,12-40,60$ & \\
\hline R-R (ms) - Média $\pm D P$ & $778,42 \pm 118,78$ & $862,57 \pm 180,43$ & \multirow{2}{*}{$0,04^{a *}$} \\
\hline IC $95 \%$ & $721,16-835,67$ & $775,61-949,54$ & \\
\hline LF/HF - Média $\pm \mathrm{DP}$ & $1,35 \pm 1,88$ & $2,48 \pm 3,64$ & \multirow{2}{*}{0,12} \\
\hline IC 95\% & $0,44-2,25$ & $0,73-4,24$ & \\
\hline Associação AAS - R-R & - & - & $0,77^{\mathrm{b}}$ \\
\hline Associação AAS - LF/HF & - & - & $0,16^{\mathrm{b}}$ \\
\hline
\end{tabular}

${ }^{a}$ Teste Wilcoxon; *p<0,05; IC - intervalo de confiança; R-R - variável R a R; Relação LF/HF; LF - Frequência baixa; HF - frequência alta. 'Teste de Sparman's $\mathrm{p}<0,05$. Fonte: Autores (2020).

Em relação aos resultados da etapa qualitativa, ou seja, dos relatos do grupo focal, após os passos da análise de conteúdo, chegou-se a duas categorias temáticas: contribuições da EOT para a promoção da saúde e contribuições da EOT para a construção de rede de apoio social.

\section{Contribuições dos EOT para a Promoção da saúde}

Este estudo revelou que a EOT contribuiu para a promoção da saúde. Percebe-se nos relatos a construção da autonomia dos participantes ao adotarem comportamentos mais saudáveis relacionados aos hábitos de vida, após iniciarem a prática da EOT, como alimentação saudável, sono mais restaurador, técnicas de respiração e meditação. 
"Eu aprendi a lidar com o sono, o fulano (instrutor) falava sobre o sono... tinha vez que eu dormia pouco tempo, muito tempo ou médio tempo, eu ia vendo como que o corpo reagia com a quantidade de sono que eu tinha. Eu comecei a perceber qual era o tempo de sono que eu acordava disposto..." Calêndula

"Eu tinha que dar preferência a escolhas saudáveis e o fulano (instrutor) falava "você tem que reduzir o açúcar, ... pelo menos destinar uma hora do dia para cuidar de nós, fazer uma meditação, nem que seja assistir uma série, conversar com outra pessoa..." Hortelã

"O fato de acompanhar a respiração, ajudou na ansiedade, na hora de apresentar trabalho, na hora da prova..." Dália

Além das mudanças dos hábitos de vida, os relatos indicaram a redução do estresse e da ansiedade com sensação de relaxamento e leveza após a prática da EOT, com destaque para as técnicas de yoga e de massagem.

“...Depois de um tempo com a prática eu saia muito leve... saia levitando, é uma prática tão gostosa que relaxa muito a gente... essa questão do estresse me ajudou, e num contexto de ansiedade, crise de pânico pude absorver e lidar comigo... na crise o que eu posso fazer... sou muito acelerada saio bem calma...” Alecrim

"Eu compartilho desse sentimento de leveza não só física como mental... o nosso contexto de o quanto a graduação exige da gente, a gente vê na prática os resultados, tem pessoas que não conseguem terminar a graduação do tanto que ela exige da nossa saúde mental e os exercícios orientais terapêuticos é uma prática que ajudou não somente todo mundo aqui, mas de uma maneira geral, completa, emocional, quanto na saúde psicológica, que é algo tão necessário ter mais sensibilidade, ter mais medidas assim na graduação..." Calêndula

"E o fulano (instrutor) acompanhava as necessidades, no fim do período ele sempre dava práticas mais relaxantes... de fazer massagem um no outro, e com temas abordando ansiedade e posturas (de yoga) que a gente podia fazer quando estivesse ansioso para prova ou outro resultado..." Camomila

Destaca-se ainda nos discursos a utilização do do-in para si e para outras pessoas fora do ambiente universitário.

“...às vezes eu estou resfriado e aperto pontos para desentupir e desentope ao longo do tempo... Eu chego na minha família e faço, o pessoal: ah, não é possivel!... Eu dizia a dor vai virar conforto. Depois diziam "faz aquele ponto comigo"'.. o ponto me ajuda, então eu comecei a fazer com os outros também, começou a ajudar as outras pessoas..." Hortelã

“Às vezes tem alguém com o nariz entupido pera aí deixa eu apertar um ponto..." Lanterna-chinesa

Logo, os relatos demonstraram que a EOT proporcionou significativas contribuições para o autoconhecimento, no reconhecimento de sentimentos, emoções e sintomas físicos diante de situações estressantes, tanto na vida pessoal, como na vida acadêmica:

“... Ter mais controle emocional principalmente na questão da raiva, naquele momento que você vai explodir e de repente fala um pouco mais rápido com alguém, tentar controlar um pouco isso, acho que hoje em dia eu consigo segurar mais... Identificar alguns gatilhos, muita coisa que eu fazia e não me faz bem, eu não conseguia perceber que me fazia mal..." Bálsamo

"Às vezes a gente escolhe algo que é o prazer imediato e não o que faz necessariamente bem. Um ponto que é verdade, é de que a dor se torna conforto. Isso para mim significa que o processo de cura não é pra ser fácil, as vezes 
cuidar de si mesmo não é uma coisa que vai ser agradável no momento, mas que a gente tem um resultado muito bom...” Erva Cidreira

"Ajuda muito no autoconhecimento, quando eu sentia dor de cabeça eu achava que era uma dor de cabeça, hoje eu já consigo ver... quando dói no meio da sobrancelha é porque está relacionado ao seu estômago. A gente vai se tocando e se auto conhecendo, percebendo como o corpo da gente funciona, também para saber lidar quando a gente passa por algum momento dificil, ansiedade e estresse, e ajuda muito a ficar bem”. Camomila

Do mesmo modo, foi possível identificar nos relatos a transformação na percepção de saúde e corpo dos estudantes da pesquisa. Além da percepção da integralidade abordada pela EOT, a compreensão oriental sobre os fenômenos e o uso de múltiplas técnicas orientais que auxiliam na prevenção e na promoção da saúde.

"É totalmente completo e com certeza fez muita diferença na minha vida, não tem nem comparação o que eu era antes do que eu sou agora..." Lanterna-chinesa

“É algo que transforma, que ajuda mesmo, tem validade aquilo... ”Calêndula

“Entrei pensando em atividade física só que depois eu vi que ia muito além, entrava na medicina tradicional chinesa, ponto de acupuntura, aí ele começava a falar de sentimentos, sobre como a gente vai reagir, como a gente consegue reagir melhor frente a problemas que a gente encontra na faculdade ou fora da faculdade”. Jasmim-manga

“É tudo muito integrado, é um exercício e alimentação, são práticas tipo ponto de acupuntura, o do-in, a yoga”.

\section{Alecrim}

“Alimentos que a gente podia consumir quando a gente está com cada sentimento, para ajudar aliviar e a ter controle, foi envolvendo muito mais do que a atividade física, envolvia alimentação, sentimento, atividade física, então é uma aula bem completa”. Jasmim-manga

\section{Contribuições da EOT para a construção de rede de apoio social}

Além das contribuições para a promoção da saúde, foi possível verificar as contribuições da EOT acerca da construção da rede de apoio social entre os participantes. Identifica-se nos discursos a presença dos vínculos desenvolvidos a partir da EOT.

“...eu tinha muito medo de pessoas, então um ponto inicial foi os exercícios orientais terapêuticos que eu comecei a conhecer mais pessoas que já estavam em outras atividades. Então eu comecei a participar de várias atividades e eu ampliei a rede...” Hortelã

“...tem a questão do vínculo e era um lugar que eu me sentia bem não só pela prática dos exercícios orientais, mas de todo mundo que ia e era um espaço que eu me sentia bem então eu acho que a questão do vínculo me ajudou a não desistir de ir nas aulas..." Girassol

“...só o que você precisava quando você estava longe da família, pelo menos eu estou longe da família e eu sou muito apegada, então no fim, na sexta só o que eu precisava era de um abraço...” Magnólia

"Você sente acolhido, confortável no lugar, então além de ser um ambiente que te distraia dessas questões estressantes da faculdade, você aprende coisas novas, você se constrói coisas novas... Então não é simplesmente se desligar, é você ligar um outro campo que talvez você esteja deixando de lado...”. Erva Cidreira 
A partir dos discursos percebe-se que a postura do instrutor da EOT foi crucial para o impacto positivo na vida dos estudantes. Os relatos indicam que ele demonstra acolhimento, empatia, habilidade para ensinar, modo leve de lidar com a vida, calma e sentem credibilidade na sua fala e pertencentes ao grupo.

"Ele tem uma forma de conduzir aula que me agrada muito... aquela calma que ele passa, consegue manter a turma atenta, sem dispersar, sem falar alto..." Bálsamo

“...Ele leva a vida de uma maneira muito leve, muito saudável ... ele foi um dos elementos que me proporcionou uma maneira de levar a vida mais suave e eu via muito isso na postura dele (professor), de levar numa boa de chamar atenção sem precisar ficar bravo.... Como foi uma prática que ele mesmo criou, adaptou, ele tinha um envolvimento..." Calêndula

"O fulano (instrutor) faz a gente sentir credibilidade nele..." Alecrim

"A linguagem, olhar para um contexto, ele consegue fazer com que a gente consiga colocar aquilo na vida..."

\section{Girassol}

"Porque a partir do momento que ele faz exercício ele fala porque que a gente está fazendo aquele exercício. Hoje eu já consigo compreender, ele nem precisa falar qual é o tema da... então deve ser uma aula que será relacionada sobre isso e sobre aquilo, então eu já começo a conectar..." Hortelã

\section{Discussão}

A pesquisa possibilitou verificar as contribuições da EOT para a promoção da saúde e para construção da rede de apoio social entre os estudantes. Foi possível verificar também que a intervenção impactou no aumento da VFC após a intervenção, identificado pelo aumento significativo da variável $R-R(p=0,04)$. A VFC permite compreender a atuação do SNA no coração, visto que representa as oscilações dos intervalos entre os batimentos cardíacos consecutivos (intervalos entre duas ondas R do eletrocardiograma, representada por R-R) no Domínio do Tempo e a atividade parassimpática (HF) e simpática (LF) no Domínio da Frequência (Catai et al., 2020).

A VFC alta indica respostas mais rápidas e melhor adaptação fisiológica frente às necessidades do organismo, principalmente a estímulos estressores, o que caracteriza um indivíduo saudável. Reduções da VFC podem refletir a diminuição da atividade vagal com prevalência da atividade simpática, além de indicar possíveis alterações fisiopatológicas (Lopes, 2014; Catai et al., 2020).

A meditação auxilia na redução da atividade simpática e aumento da parassimpática (Kim et al., 2017). A prática de yoga e meditação, por meio do aumento da VFC indica atuação autonômica satisfatória, além da redução dos níveis de estresse e melhora do sono (Wolever et al., 2012). O predomínio da estimulação parassimpática pode indicar potencial relaxamento dos participantes após a prática realizada nesse estudo.

A Yoga é considerada uma prática de origem oriental utilizada para o controle do corpo e mente. A meditação, também originada a partir de filosofias orientais, compreende uma prática mental e individual que busca o treino do foco a diminuir os pensamentos repetitivos e promover melhora do desempenho cognitivo com maior integração entre mente, corpo e ambiente externo (Brasil, 2018).

Segundo o instrutor e idealizador da EOT:

"Foi possivel transpor as posições de yoga para os sistemas teóricos e práticos da medicina chinesa, principalmente para a teoria dos cinco elementos. Se uma pessoa tem um desequilíbrio no elemento madeira, por exemplo, podemos utilizar posturas que beneficiam o fígado, e consequentemente, contribuir para o equilíbrio da madeira. Esse tipo de 
aplicação pode ser utilizado para todos os órgãos e vísceras da medicina chinesa, mapeando as posturas que beneficiam essas estruturas".

Embora esse estudo tenha observado um aumento entre os intervalos R-R, não apresentou redução significativa da relação LF/HF. A razão LF/HF é considerada por alguns investigadores o equilíbrio simpático/vagal ou a manifestação da atividade simpática (Catai et al., 2020). Em discordância, a prática de yoga, em outro estudo obteve redução expressiva da relação LF/HF se refletindo na maior atividade parassimpática e equílibrio simpático-vagal (Bhaskar et al., 2017)

Tendo em vista que a secreção de AAS é um importante biomarcador para avaliar intervenções que buscam a melhoria da saúde dos sujeitos frente ao estresse (Ali \& Nater, 2020), nesse estudo essa variável não sofreu impacto da intervenção, visto que antes e após a EOT, a média dos valores de AAS (menor que $34 \mathrm{KU} / \mathrm{L}$ ), não indicava predomínio de atividade simpática, ou seja, presença de estresse na população estudada.

Coaduna-se com o estudo de Moura et al. (2018), que obteve média de $31 \mathrm{KU} / \mathrm{L}$ da AAS e concluiu que os resultados da AAS não foram semelhantes aos achados de ansiedade e de depressão avaliados por escalas e pelo auto relato dos estudantes universitários. Dessa maneira, a falta de relação da AAS com o relato verbal pode sugerir que o aparelho utilizado não seja o mais adequado para a mensuração de AAS na população universitária.

Em relação a promoção da saúde, um dos aspectos fundamentais é a construção da autonomia. Seu estímulo potencializa a saúde dos sujeitos e envolve transformações profundas para uma boa saúde (Czeresnia \& Freitas, 2020). Na visão chinesa a "boa saúde" é pautada não só na harmônica circulação do Qí pelos meridianos, mas no equilíbrio da vitalidade e no desenvolvimento da consciência corporal por um processo sereno e introspectivo (Coutinho \& Dulcetti, 2015; Contatore et al., 2018).

Destaca-se o potencial da universidade como agente promotor da saúde, principalmente em relação às ações de extensão que podem contribuir para a educação em saúde, ampliação do autocuidado, auto percepção e bem-estar (Fonseca et al., 2020). Posto isto, salienta-se a responsabilidade das instituições de ensino superior (IES) em desenvolver estratégias que promovam o desenvolvimento integral dos seus estudantes. Além disso, recomendam-se práticas de meditação, de relaxamento e de atividade física para redução do estresse, na promoção da saúde e na prevenção de agravos dessa população (Costa et al., 2020).

Ressalta-se que essas ações de extensão universitária se configuram como ferramentas imprescindíveis dentro do ensino superior, contribuindo para a superação do paradigma biomédico curativista, além de incentivarem o desenvolvimento do potencial dos sujeitos como promotores da sua saúde e compartilhadores dos conhecimentos apreendidos (Fonseca et al., 2020). Adicionalmente, fomentam o estímulo a mudanças de comportamento em relação aos hábitos de vida, incentivam o autocuidado e o autoconhecimento para a saúde (Czeresnia \& Freitas, 2020; Fonseca et al., 2020).

É oportuno assinalar que o do-in busca prevenir e tratar distúrbios no corpo pela restauração do fluxo da energia. Embora o do-in ou acupressão compartilhem aspectos em comum com a técnica de massagem shiatsu, são diferentes quanto a sua filosofia e técnica (Brasil, 2018; Cabo et al., 2018). Para compreender a eficácia verdadeira do shiatsu, Cabo et al. (2018) apontam a necessidade de uma definição comum sobre a prática na literatura, tendo em vista que as evidências têm utilizado shiatsu e acupressão de forma análoga. Segundo esses autores, o do-in é uma técnica chinesa que utiliza a acupressão nos pontos correspondentes aos meridianos energéticos do corpo utilizados na acupuntura. A técnica de massagem utilizada, Shiatsu, utiliza-se desta em diferentes partes do corpo fazendo pressão com os dedos e palmas das mãos com a utilização do peso do corpo de quem a está aplicando (Brasil, 2018; Cabo et al., 2018). 
Haja vista que o paradigma biomédico hegemônico suprimiu o processo existente entre causa e efeito no processo saúde-doença por meio da consolidação do pensamento dualista normal ou patológico (Coutinho \& Dulcetti, 2015). Diante de tais apontamentos é imprescindível destacar a diferença existente entre a medicina ocidental e a medicina tradicional chinesa.

Os katis, também utilizados na EOT, derivam do Kung Fu-Wushu. Na China, a palavra Wushu denomina o conjunto de conhecimentos relacionados às artes marciais. No Ocidente, o conceito Kung $F u$ denomina todas as modalidades de artes marciais de origem chinesa. Os Katis designam uma sequência de movimentos individuais de ataque e defesa aliados à resistência física, à força, à velocidade, ao equilíbrio e à elasticidade. São movimentos corporais harmônicos sincronizados à respiração (Rocha, 2005).

O estudo realizado por Cintra e Pereira (2012) evidenciou que o contato com a ótica chinesa possibilitou às pessoas do seu estudo conhecer uma concepção de corpo diferente da apresentada pelo modelo biomédico. Novas explicações para a relação de causa e efeito entre corpo/mente/emoção/sintomas promove transformações em formas de cuidado com o corpo.

Nesse sentido, a promoção da saúde pela medicina chinesa só é possível através da compreensão e utilização dos fundamentos de sua cosmologia. Quando as técnicas da MTC são implementadas através da ótica biomédica, o tratamento se torna restrito apenas aos sinais e sintomas, no sentido de suprimi-los e não singularizá-los, diferentemente da compreensão da interação do movimento Yin e Yang na vida de cada sujeito (Coutinho \& Dulcetti, 2015).

Tendo em vista as contribuições da EOT para a construção da rede social de apoio, para que a adaptação à universidade seja satisfatória, é necessário um repertório de habilidades sociais nas relações interpessoais, capazes de auxiliar no enfrentamento das adversidades do meio acadêmico. O melhor desempenho interpessoal dos discentes que vivem fora de suas residências e longe da família advém do estabelecimento de relações de apoio e suporte social com os colegas (Soares \& Prette, 2015).

Dessa maneira, compreende-se rede de apoio social como uma teia de relações interligando os sujeitos que possuem vínculos entre si, possibilitando que os recursos de apoio fluam entre os vínculos (Bowling, 2003). Construir e consolidar as redes é um processo ligado a convivência. Na ausência das redes, verifica-se o aumento da vulnerabilidade dos sujeitos diante de uma situação de risco (Juliano \& Yunes, 2014). No entanto, ressalta-se que a qualidade das relações estabelecidas entre os vínculos é crucial para o desenvolvimento de experiências positivas e para que a rede seja considerada como um fator protetivo (Nunes et al., 2020)

Czeresnia \& Freitas (2020) assinalam que o conhecimento sobre a epidemiologia é fundamental no desenvolvimento de práticas de saúde pública. Nesta direção, para promoção da saúde e prevenção da ideação suicida, enquanto problema de saúde pública prevalente na população universitária, torna-se essencial o desenvolvimento de intervenções no contexto universitário (Santos et al., 2017).

Tanto as vivências da transição para o ensino superior, quanto para o mercado de trabalho, são momentos tangenciados por angústias, incertezas e inseguranças. Por isso, o apoio social é fundamental para os universitários às adaptações exigidas pelo meio formativo. Geralmente, os estudantes que se declaram mais satisfeitos e com melhor autoestima se percebem mais apoiados socialmente e estabelecem interações sociais mais positivas (Matias \& Martinelli, 2017).

Nesse sentido, ações de promoção da saúde com foco no pertencimento a um grupo, sensação de autoconfiança e a harmonia com o meio social, tendem a valorizar o resgate da autoestima e a adaptação ao meio. Entretanto, essas ações podem desconectar a pessoa do seu contexto sociocultural e fazer com que se sinta responsabilizada por seguir um estilo de vida saudável. Sendo assim, uma educação emancipadora propõe ações dialógicas de acordo com o contexto vivenciado pelas pessoas, valoriza os seus saberes, constrói parcerias, bem como, autonomias responsáveis e solidárias (Tesser, 2009). 
Outro aspecto importante é a postura do instrutor da EOT. Desse modo, observa-se que o professor modelo é aquele que serve de exemplo aos estudantes por suas qualidades ao demonstrar habilidades e características pessoais que inspirem, como a empatia, a habilidade para ensinar e o profissionalismo (Silva et al., 2019).

Portanto, tendo em vista as contribuições gerais da EOT, Contatore et al. (2018) discorrem sobre a impossibilidade sociológica de validar as medicinas complementares, dado que o processo de validação científica é baseado num modelo de produção de evidência ocidental e em noções calcadas na ciência biomédica, num processo de colonização ocidental dos saberes orientais. Por isso, certamente, trata-se de uma temática atual necessária de ser explorada para que haja o reconhecimento das PICs como um eixo fundamental na redefinição do modelo de atenção em saúde como forma de questionar a hegemonia do paradigma biomédico atual (Silva et al., 2020).

Dessa maneira, torna-se importante destacar as contribuições significativas da EOT na vida dos estudantes dessa pesquisa e valorizar saberes orientais milenares como forma de ampliar a compreensão e o cuidado dos sujeitos e da coletividade. Essas contribuições só foram possíveis de serem compreendidas na etapa qualitativa desse estudo, portanto, salienta-se a importância da utilização desse método para conhecer o impacto subjetivo das práticas complementares na vida das pessoas.

\section{Considerações Finais}

Constatou-se que a EOT contribuiu para a promoção da saúde ao reduzir o estresse, a ansiedade e ao produzir relaxamento e leveza como sensação predominante após a prática; implicou em maior controle emocional, autoconhecimento e autonomia. Adicionalmente, verificou-se que ao valorizar e ao compreender os fundamentos cosmológicos orientais impactou significativamente a vida dos estudantes dessa pesquisa, bem como, possibilitou ampliar a compreensão do cuidado aos sujeitos e à coletividade. Destaca-se ainda, a importância da EOT na ampliação da rede social dos estudantes e no sentimento de pertencimento ao grupo.

No que tange aos parâmetros fisiológicos averiguou-se que os estudantes possivelmente não estavam estressados, visto que a média de AAS foi abaixo de $34 \mathrm{KU} / \mathrm{L}$. Evidenciou-se que a EOT influenciou no aumento da VFC, ou seja, auxiliou na redução da FC e no relaxamento. Entretanto, não houve associação entre a VFC e AAS. Em relação às limitações do estudo, não houve grupo controle e a coleta de dados foi transversal. Sugere-se estudos longitudinais, cálculo amostral e a exclusão de participantes sem alterações na AAS que indicam estresse.

Fomenta-se a adoção de estratégias minimizadoras de agravos e de promoção da saúde no ambiente acadêmico, uma vez que a EOT é considerada um método inovador ao articular terapias complementares existentes numa só atividade norteada pela integralidade e necessidades ampliadas de saúde dos estudantes universitários.

\section{Agradecimentos}

À Pró-reitoria de Extensão da Universidade Federal de Alfenas (UNIFAL-MG) pelo subsídio na execução das ações do Programa Unifal Sem Estresse (PULSE).

\section{Referências}

Ali, N., \& Nater, U. M. (2020). Salivary Alpha-Amylase as a Biomarker of Stress in Behavioral Medicine. International Journal of Behavioral Medicine, 27(3), 337-342.

Andifes. (2019). V Pesquisa do Perfil Socioeconômico e Cultural dos Estudantes de Graduação. Uberlândia.

Bardin, L. (2016). Análise de conteúdo: edição revista e ampliada. Edições 70. 
Bhaskar, L., Kharya, C., Deepak, K. k., \& Kochupillai, V. (2017). Assessment of Cardiac Autonomic Tone Following Long Sudarshan Kriya Yoga in Art of Living Practitioners. The Journal of Alternative and Complementary Medicine, 23(9), 705-712.

Brasil. (2006). Aprova a Política Nacional de Práticas Integrativas e Complementares (PNPIC) no Sistema Único de Saúde. Portaria $n^{\circ} 971$, de 03 de maio de 2006. https://bvsms.saude.gov.br/bvs/saudelegis/gm/2006/prt0971_03_05_2006.html

Brasil. (2018). Glossário Temático: Práticas Integrativas e Complementares em Saúde. Ministério da Saúde.

Bowling, A. (2004). Measuring health. A review of quality of life measurement scales. (3a ed.).

Cabo, F., Baskwill, A., Aguaristi, I., Christophe-Tchakaloff, S., \& Guichard, J. P. (2018). Shiatsu and acupressure: Two different and distinct techniques. International Journal of Therapeutic Massage and Bodywork: Research, Education, and Practice, 11(2), 4-10.

Catai, A. M., Pastre, C. M., Godoy, M. F. de, Silva, E. da, Takahashi, A. C. de M., \& Vanderlei, L. C. M. (2020). Heart rate variability: Are you using it properly? Standardisation checklist of procedures. Brazilian Journal of Physical Therapy, 24(2), 91-102.

Cintra, M. E. R., \& Pereira, P. P. G. (2012). Percepções de corpo identificadas entre pacientes e profissionais de medicina tradicional chinesa do Centro de Saúde Escola do Butantã. Saúde e Sociedade, 21(1), 193-205.

Contatore, O. A., Tesser, C. D., \& Barros, N. F. (2018). Medicina chinesa/acupuntura: Apontamentos históricos sobre a colonização de um saber. História, Ciências, Saúde-Manguinhos, 25(3), 841-858.

Costa, D. S. da, Medeiros, N. de S. B., Cordeiro, R. A., Frutuoso, E. de S., Lopes, J. M., \& Moreira, S. da N. T. (2020). Sintomas de Depressão, Ansiedade e Estresse em Estudantes de Medicina e Estratégias Institucionais de Enfrentamento. Revista Brasileira de Educação Médica, 44(1).

Coutinho, B. D., \& Dulcetti, P. G. S. (2015). O movimento Yīn e Yáng na cosmologia da medicina chinesa. História, Ciências, Saúde-Manguinhos, 22(3), $797-811$.

Creswell, J. W., \& Plano Clark, V. (2018). Designing and conducting mixed methods research. SAGE.

Czeresnia, D., \& Freitas, CM. (2020). Promoção da Saúde: conceitos, reflexões, tendências: Editora Fiocruz.

Fonseca, Y. S., Manganelli, L. A. G., Borges, G. F., Alves, C. O., Barbosa, G. de A., Correia, A. P., \& Mascarenhas, A. G. (2020). Ação extensionista de promoção da saúde de estudantes universitários. Extensio: Revista Eletrônica de Extensão, 17(35), 81-95.

Juliano, M. C. C., \& Yunes, M. A. M. (2014). Reflexões sobre rede de apoio social como mecanismo de proteção e promoção de resiliência. Ambiente \& Sociedade, 17(3), 135-154.

Kim, B. J., Cho, I. S., \& Cho, K. I. (2017). Impact of Mindfulness Based Stress Reduction Therapy on Myocardial Function and Endothelial Dysfunction in Female Patients with Microvascular Angina. Journal of Cardiovascular Ultrasound, 25(4), 118-123.

Kinalski, D. D. F., Paula, C. C., Padoin, S. M. de M., Neves, E. T., Kleinubing, R. E., \& Cortes, L. F. (2017). Focus group on qualitative research: Experience report. Revista Brasileira de Enfermagem, 70(2), 424-429.

Lopes, P., Oliveira, M., André, S., Nascimento, D., Silva, C., Rebouças, G., Felipe, T., Albuquerque-Filho, N., \& Medeiros, H. (2014). Aplicabilidade Clínica da Variabilidade da Frequência Cardíaca. Revista Neurociências, 21(04), 600-603.

Matias, R. de C., \& Martinelli, S. de C. (2017). Um estudo correlacional entre apoio social e autoconceito de estudantes universitários. Avaliação: Revista da Avaliação da Educação Superior (Campinas), 22(1), 15-33.

McEwen, B. S. (2007). Physiology and Neurobiology of Stress and Adaptation: Central Role of the Brain. Physiological Reviews, 87(3), 873-904.

Minayo, M. C. S. (2017). Amostragem e saturação em pesquisa qualitativa: consensos e controvérsias. Revista Pesquisa Qualitativa, 5(7), 1-12.

Nascimento, M. C. do, Barros, N. F. de, Nogueira, M. I., \& Luz, M. T. (2013). A categoria racionalidade médica e uma nova epistemologia em saúde. Ciência \& Saúde Coletiva, 18(12), 3595-3604.

Nunes, T. G. R., Pontes, F. A. R., \& Silva, L. I. da C. (2020). Juventude e apoio social: Um olhar sobre as redes sociais de estudantes paraenses. Práxis Educativa (Brasil), 15, 01-21.

Oliveira, E. S. de, Silva, A. F. R. da, Silva, K. C. B. da, Moura, T. V. C., Araújo, A. L. de, \& Silva, A. R. V. da. (2020). Stress and health risk behaviors among university students. Revista Brasileira de Enfermagem, 73(1).

Polit, D. F., \& Beck, C. T. (2018). Fundamentos de Pesquisa em Enfermagem: Avaliação de Evidências para a Prática da Enfermagem. Porto Alegre: Artmed.

Resende, 1. C. (2019). Programa "UNIFAL-MG Sem Estresse" realiza ações para promoção da saúde; confira cronograma das atividades na Sede e nos campi. https://www.unifal-mg.edu.br/portal/2019/05/23/programa-unifal-mg-sem-estresse-realiza-acoes-para-promocao-da-saude-confira-cronograma-das-atividadesna-sede-e-nos-campi/

Rocha, L. G. F. (2005). Estratégias de ensino-aprendizagem do Kung-Fu para pessoas com deficiência visual. Trabalho de Conclusão de Curso (TCC) na Educação Física - UNICAMP, São Paulo.

Santos, H. G. B. dos, Marcon, S. R., Espinosa, M. M., Baptista, M. N., \& Paulo, P. M. C. de. (2017). Factors associated with suicidal ideation among university students. Revista Latino-Americana de Enfermagem, 25. 
Research, Society and Development, v. 10, n. 4, e46810413481, 2021

(CC BY 4.0) | ISSN 2525-3409 | DOI: http://dx.doi.org/10.33448/rsd-v10i4.13481

Shetty, V., Zigler, C., Robles, T. F., Elashoff, D., \& Yamaguchi, M. (2011). . Developmental validation of a point-of-care, salivary $\alpha$-amylase biosensor. Psychoneuroendocrinology, 36(2), 193-199.

Silva, G. K. F. da, Sousa, I. M. C. de, Cabral, M. E. G. da S., Bezerra, A. F. B., \& Guimarães, M. B. L. (2020). Política Nacional de Práticas Integrativas e Complementares: Trajetória e desafios em 30 anos do SUS. Physis: Revista de Saúde Coletiva, 30(1).

Soares, A. B., \& Prette, Z. A. P. D. (2015). Habilidades sociais e adaptação à universidade: Convergências e divergências dos construtos. Análise Psicológica, 33(2), 139-151.

Tesser, C. D. (2009). Práticas complementares, racionalidades médicas e promoção da saúde: Contribuições poucos exploradas. Cadernos de Saúde Pública, $25(8), 1732-1742$.

User Manual for Polar V88 in English. (2019). https://support.polar.com/en/support/V800/User_manual_for_polar_v800_in_english

Wen, T. S. (2008). Manual Terapêutico de Acupuntura. Manole.

Wolever, R. Q., Bobinet, K. J., McCabe, K., Mackenzie, E. R., Fekete, E., Kusnick, C. A., \& Baime, M. (2012). Effective and viable mind-body stress reduction in the workplace: A randomized controlled trial. Journal of Occupational Health Psychology, 17(2), 246-258.

Yamaguchi, M., Kanemori, T., Kanemaru, M., Takai, N., Mizuno, Y., \& Yoshida, H. (2004). Performance evaluation of salivary amylase activity monitor. Biosensors and Bioelectronics, 20(3), 491-497. 